\title{
OPEN Homocysteine level is positively and independently associated with serum creatinine and urea nitrogen levels in old male patients with hypertension
}

\author{
Qianhong Yang ${ }^{\bowtie}$, Youwei Lu, Yanhua Deng, Jiayi Xu \& Xi Zhang
}

A cross-sectional study to show whether and how serum fasting homocysteine levels are associated with renal function changes in patients with hypertension. Homocysteine levels were associated with serum creatinine and blood urea nitrogen (BUN) levels with coefficients of 2.04 and 0.07 , respectively, only in males and independent of confounders. In addition, low density lipoprotein cholesterol (LDL-C) levels were positively and left ventricular ejection fraction (LVEF) was negatively associated with serum creatinine level in males; age was positively associated with serum creatinine levels in females. Age was a common risk factor positively associated with BUN levels in both sexes, while total cholesterol (TC) levels and glycemic control were independent risk factors that were positively associated with BUN levels only in males. LDL-C levels and LVEF were negatively associated with BUN levels in females. Body mass index (BMI) was positively associated and hemoglobin A1c (HbA1c) levels, high density lipoprotein cholesterol (HDL-C) levels and the presence of stroke were negatively associated with serum uric acid levels in male patients. In contrast, only LVEF was positively associated with uric acid levels in females. In conclusion, homocysteine level is an independent risk factor associated with serum creatinine and BUN levels in male patients with hypertension.

Homocysteine is a sulfur-containing, nonproteinogenic amino acid that is derived from transmethylation of the essential sulfur-containing amino acid methionine ${ }^{1}$. Homocysteine can be either degraded through the transsulfuration pathway or remethylated to methionine $e^{2,3}$. In the transsulfuration pathway, homocysteine combines with serine to form cystathionine; then, cystathionine is hydrolyzed into cysteine and alpha-ketobutyrate ${ }^{2}$. There are two different remethylation pathways: the first remethylation pathway requires 5-methyltetrahydrofolate as a methyl donor, which is generated by a reaction catalyzed by 5,10-methylenetetrahydrofolate reductase (MTHFR); the second remethylation reaction uses betaine as a methyl donor in the transsulfuration pathway ${ }^{2,3}$. Both remethylation reactions are irreversible and require the active form of $\mathrm{B}$ vitamins as a cofactor ${ }^{4,5}$. Homocysteine is filtered by the glomerulus; however, it is almost completely (over 98\%) reabsorbed by tubular cells in the kidneys ${ }^{3}$.

The normal total fasting plasma homocysteine levels range from 4 to $12.3 \mu \mathrm{mol} / \mathrm{l}^{6}$. Hyperhomocysteinemia is currently defined as a homocysteine level above $10 \mu \mathrm{mol} / \mathrm{l}$ in the blood and is demonstrated as a pathological condition ${ }^{6,7}$. Currently, the hypothesis focused on homocysteine toxicity can be summarized as homocysteinylation (protein structure modifications); oxidative stress induction; and excitotoxicity ${ }^{1}$. These biotoxicities are believed to act in numerous diseases ${ }^{1}$. Hyperhomocysteinemia has been recognized to be associated with cardiovascular diseases $(\mathrm{CVDs})^{8}$, neurological and psychiatric disorders ${ }^{9}$, bone tissue damage ${ }^{10}$, gastrointestinal disorders ${ }^{11}$, cancer $^{12}$, and congenital disorders as well as chronic kidney disease $(C K D)^{1,13}$. In addition, the prevalence of hyperhomocysteinemia is high in patients undergoing peritoneal dialysis and in renal transplantation recipients ${ }^{4}$.

The existence of a narrow crosstalk between homocysteine, hypertension and renal disease in pathophysiological processes has increased the difficulty of research attempting to identify the pathogenesis of hyperhomocysteinemia. Homocysteine is an independent risk factor for both hypertension and CKD; at the same time, hypertension is also a well-documented cause of $\mathrm{CKD}^{2}$. However, studies focused on the correlations between 

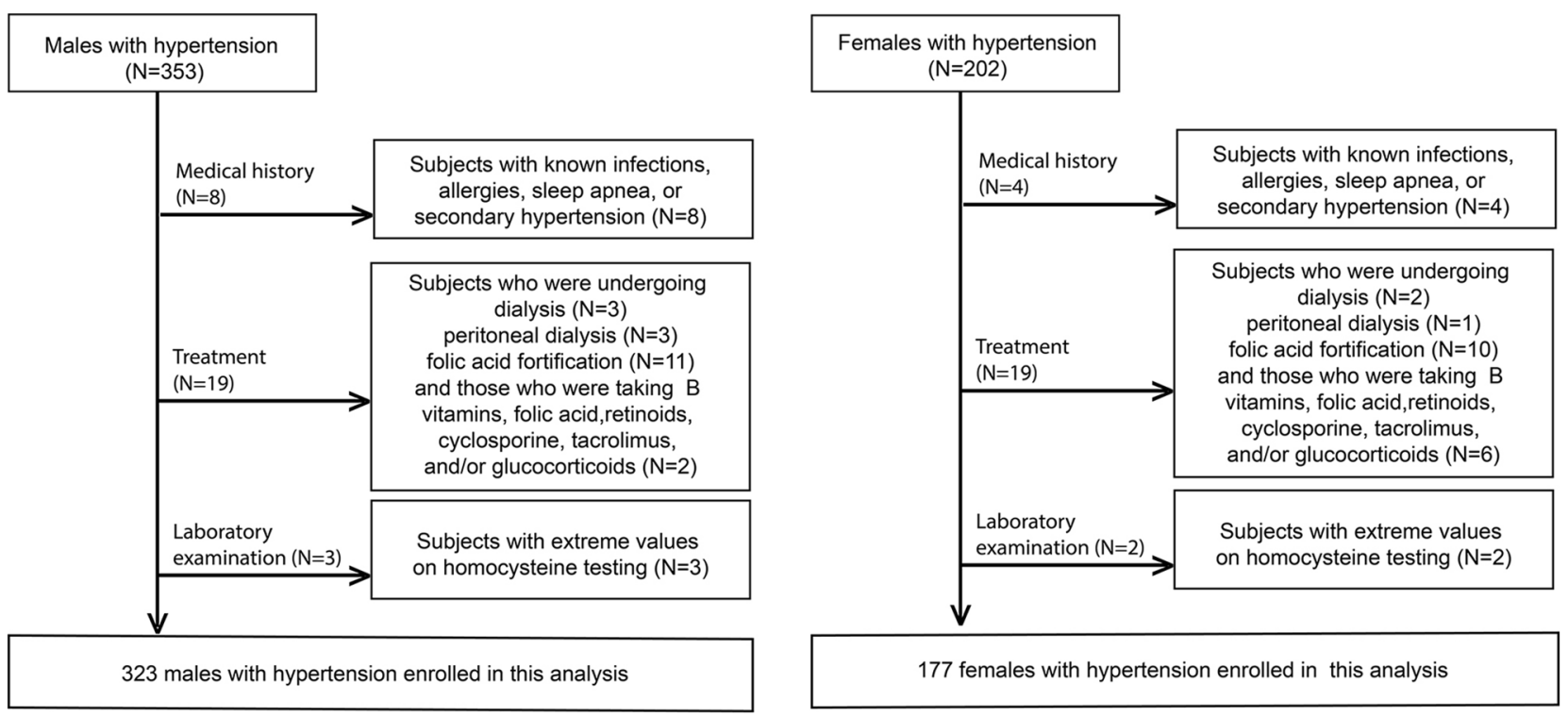

Figure 1. Flow chart of participants. In total, 353 males and 202 females were involved initially. 30 males and 25 females were excluded based on exclusion criteria. Finally, 323 males and 177 females were involved in this study.

homocysteine levels and renal disorders remain scarce ${ }^{14}$, and the existing research differs in the study design, objective, setting and participants. Although observational studies have shown that hyperhomocysteinemia is associated with the risk of developing CKD and albuminuria in the general population ${ }^{5,14-17}$, reports showed that elevated serum homocysteine levels are not correlated with serum uric acid levels in patients with gout ${ }^{18}$.

China is a country where no folic acid fortification is implemented. Hypertension and hyperhomocysteinemia often presented together (termed $\mathrm{H}$-type hypertension), which accounts for approximately $75 \%$ of patients with hypertension ${ }^{19-21}$. Chinese clinicians believe that $\mathrm{H}$-type hypertension is correlated with a higher occurrence of stroke in Chinese adults with hypertension ${ }^{22}$. However, how serum homocysteine levels are associated with renal function in Chinese patients with hypertension remains unclear. In this report, this issue was evaluated in 323 male and 177 female hypertension patients.

\section{Methods}

Study design and participants. This is a cross-sectional study to show whether and how serum fasting homocysteine levels are associated with renal function indicators in old Chinese patients with hypertension. To derive more information from the relationship between homocysteine level and renal function, we have not used a surrogate marker eGFR and instead used serum creatinine, BUN and uric acid levels as renal function indicators directly. Initially, 353 male and 202 female registered hypertensive inpatients in the Departments of Geriatrics in our hospital in 2019 were continuously assembled. Subjects who were undergoing dialysis $(\mathrm{N}=5)$ or peritoneal dialysis $(\mathrm{N}=4)$ or folic acid fortification $(\mathrm{N}=21)$ and those who were taking $\mathrm{B}$ vitamins, folic acid, retinoids, cyclosporine, tacrolimus, and/or glucocorticoids $(\mathrm{N}=8)$ were excluded, as were those with known infections, allergies, sleep apnea, or secondary hypertension $(\mathrm{N}=12)$ and those with extreme values on homocysteine testing ( $>40.0 \mu \mathrm{mol} / \mathrm{l}, \mathrm{N}=5)$ (Fig. 1). Finally, 323 males and 177 females were involved in this study.

The study was approved by the institutional review board at Minhang Hospital, Fudan University and was conducted in full accordance with the principles of the Declaration of Helsinki. All patients provided written informed consent.

Data collection. Age, weight, height, smoking, alcohol consumption, history of hypertension, stroke, diabetes, CHD, CKD and medications were recorded using an admission registration questionnaire. Smoking was defined as smoking five or more cigarettes daily for at least 3 months. Alcohol consumption was defined as drinking up to one alcoholic drink per day for women and up to two per day for men irrespective of liquor types. Body mass index (BMI) was calculated as weight divided by height squared $\left(\mathrm{kg} / \mathrm{m}^{2}\right)$. Waking blood pressure was measured between the hours of 8 and 11 a.m. in the seated position after a 5-min rest. It was recorded as the mean of three measurements taken at 1-min intervals according to American Society of Hypertension guidelines and using a mercury sphygmomanometer. Hypertension was defined as a systolic pressure greater than $140 \mathrm{~mm}$ $\mathrm{Hg}$ or a diastolic pressure greater than $90 \mathrm{~mm} \mathrm{Hg}$ or according to medical history.

A fasting blood sample of each participant was sampled from the antecubital vein the next morning after admission. Serum lipid profiles, HCY, folic acid, HbA1c creatinine, BUN, uric acid, and serum glucose concentrations were measured by the hospital laboratory according to routine procedures. Diabetes was defined as a fasting plasma glucose of at least $7.0 \mathrm{mmol} / \mathrm{L}$ or treatment with antidiabetic medication before the measurement. 
A transthoracic echocardiographic examination was performed using a Sonos 5500 type Ultrasound machine (Philips, Best, Netherlands) with a $2.5-\mathrm{Hz}$ transducer. The measurement of the left ventricular ejection fraction was performed using Simpson's biplane method.

The diagnosis of CHD and CKD followed the 2019 ESC Guidelines for the Diagnosis and Management of Chronic Coronary Syndromes ${ }^{23}$. The Task Force for the Diagnosis and Management of Chronic Coronary Syndromes of the European Society of Cardiology (ESC) and Classification and Diagnosis of Diabetes: Standards of Medical Care in Diabetes-202024.

Statistical analysis. All variables generally fit a normal distribution as checked by histogram analysis after eliminating a small number of extremes ( $<1 \%$ for each variate). Shapiro-Wilk test for three dependent variables, serum creatinine, BUN and serum uric acid, also confirmed their normality $(p>0.05)$. Thus, they are presented as the means \pm standard deviations.

To learn whether and how serum creatinine, BUN and uric acid levels were associated with fasting serum homocysteine level, regression modeling was adopted. To eliminate the effect of multicollinearity on the results, internal correlations within parameters were quantified by calculating the Pearson correlation coefficient. The levels of serum creatinine, BUN and serum uric acid displayed high and significant correlations between each other; therefore, these variables were designated as dependent variate solely in each regression analysis. Age, BMI, levels of homocysteine, folic acid, creatinine, BUN, uric acid, TC, TG, LDL-C, and HDL-C, SBP, DBP, percentages of $\mathrm{HbAlc}$ and LVEF, presence of diabetes, CHD, CKD and stroke, and history of medication, smoking and drinking were treated as independent variates.

The associations between independent and dependent variates were evaluated by two steps, univariate and multivariate linear regressions. In univariate regression analysis, the associations between each independent variate and each dependent variate were evaluated, any independent variate that displayed $\mathrm{p}<0.10$ in a given univariate analysis model was considered as a potential independent variable.

In multivariate regression analysis, the independent variable selection was based on the preliminary univariate analysis, Pearson correlation coefficient and principles of biochemistry and physiology (detailed in the results); independent factors associated with renal function indicators were evaluated under adjustment of these confounding factors with backward selection. We considered $P<0.05$ by a two-sided test to be indicative of statistical significance. Statistical analyses were performed using SPSS ver. 17.0.0 (SPSS, Chicago, IL, USA).

\section{Results}

Participants. As summarized in Table 1, the average ages of males and females were $75.6 \pm 13.7$ and $75.9 \pm 11.3$ years, respectively. The average BMI of males and females were $24.5 \pm 3.0$ and $24.3 \pm 3.8 \mathrm{~kg} / \mathrm{m}^{2}$, respectively, suggesting that most of our subjects had a normal to overweight BMI. The average HCY levels of males and females were $16.5 \pm 7.1$ and $13.6 \pm 5.9 \mu \mathrm{mol} / \mathrm{L}$, respectively, which indicates that the HCY levels of most subjects exceeded the upper bound. The average serum folic acid levels of males and females were $7.6 \pm 4.6$ and $9.2 \pm 4.7 \mathrm{ng} / \mathrm{mL}$, which shows that most of our subjects possessed a normal range of serum folic acid levels $(2.7$ to $17.0 \mathrm{ng} / \mathrm{mL}$ ). Approximately $42.9 \%$ males and females had diabetes, and the average HbAlc levels of males and females were $6.8 \pm 1.7$ and $6.6 \pm 1.6 \%$, respectively, which is consistent with a high prevalence of diabetes among our subjects. Although only $13.4 \%$ of males and $7.3 \%$ of females were diagnosed with CKD and the average serum creatinine, BUN and uric acid levels were in the normal ranges, their renal function adjoined to the upper limits. Although all participants had hypertension, the average SBP and DBP for males and females were $138.9 \pm 23.4$ and $141.2 \pm 19.4$, respectively, and $78.2 \pm 12.0$ and $78.6 \pm 10.7 \mathrm{mmHg}$, respectively. These measures were relatively low and might be due to medication since $75.8 \%$ of males and $78.5 \%$ of females were taking betablockers, angiotensin-converting enzyme inhibitors, angiotensin receptor blockers, statins and/or antidiabetic agents.

Factors associated with serum creatinine level in the univariate regression analysis. To identify which clinical parameters are associated with renal function change, in the following analyses, serum creatinine, BUN and uric acid were treated as dependent variables, and the other parameters listed in the Table 1 treated as independent variables to perform the univariate regression analysis.

As shown in Table 2 (upper panel), serum HCY, BUN, uric acid, TC and LDL-C levels, SBP, and DBP $(P=0.074)$ were positively associated and LVEF was negatively associated with serum creatinine levels in male patients with hypertension. Age and serum HCY, BUN and uric acid levels were positively associated with serum creatinine level in female patients with hypertension; serum creatinine levels were higher in females with CHD and CKD and lower in those with stroke.

Factors associated with serum BUN level in the univariate regression analysis. As shown in Table 2 (middle panel), age and serum HCY and TC $(P=0.091)$ levels were positively associated and LVEF was negatively associated with serum BUN levels in male patients; serum BUN levels were higher in male patients with diabetes and CKD who had glycemic control; conversely, the BUN levels were lower in males with stroke $(P=0.076)$ and in males who consumed alcohol. Age and HCY levels were positively associated and LDL-C levels $(P=0.093)$, DBP $(P=0.076)$ and LVEF were negatively associated with serum BUN levels in female patients; serum BUN levels were higher in females who had glycemic control, $\mathrm{CHD}(P=0.056)$ and $\mathrm{CKD}$ and were lower in those with stroke.

Factors associated with serum uric acid level in the univariate regression analysis. As shown in Table 2 (lower panel), age $(P=0.093)$ and $\mathrm{HbAlc}$ and HDL-C levels were factors negatively associated and BMI 


\begin{tabular}{|l|l|l|}
\hline \multirow{2}{*}{ Variable } & Male & Female \\
\cline { 2 - 3 } & $\mathbf{N = 3 2 3 )}$ & $\mathbf{N}=177)$ \\
\hline Average age, years & $75.6 \pm 13.7$ & $75.9 \pm 11.3$ \\
\hline Age range, years & $40.0-99.0$ & $41.0-98.0$ \\
\hline Lower quartile, median and upper quartile of age, years & $66.0,75.0,87.0$ & $66.0,73.0,84.0$ \\
\hline BMI, kg/m ${ }^{2}$ & $24.5 \pm 3.0$ & $24.3 \pm 3.8$ \\
\hline HCY, $\mu \mathrm{mmol} / \mathrm{L}$ & $16.5 \pm 7.1$ & $13.6 \pm 5.9$ \\
\hline Folic acid, ng/mL & $7.6 \pm 4.6$ & $9.2 \pm 4.7$ \\
\hline HbAlc, \% & $6.8 \pm 1.7$ & $6.6 \pm 1.6$ \\
\hline Creatinine, $\mu \mathrm{mol} / \mathrm{L}$ & $99.2 \pm 72.1$ & $76.8 \pm 49.0$ \\
\hline BUN, mmol/L & $6.6 \pm 3.4$ & $6.0 \pm 2.9$ \\
\hline Uric acid, $\mu \mathrm{mol} / \mathrm{L}$ & $353.6 \pm 103.8$ & $312.1 \pm 104.6$ \\
\hline TC, mmol/L & $3.9 \pm 1.0$ & $4.3 \pm 1.1$ \\
\hline TG, mmol/L & $1.5 \pm 1.0$ & $1.6 \pm 1.0$ \\
\hline LDL-C, mmol/L & $2.6 \pm 0.9$ & $2.8 \pm 1.0$ \\
\hline HDL-C, mmol/L & $1.0 \pm 0.3$ & $1.2 \pm 0.3$ \\
\hline SBP, mmHg & $138.9 \pm 23.4$ & $141.2 \pm 19.4$ \\
\hline DBP, mmHg & $78.2 \pm 12.0$ & $78.6 \pm 10.7$ \\
\hline LVEF, \% & $62.9 \pm 5.1$ & $62.6 \pm 5.0$ \\
\hline Presence of diabetes, cases (\%) & $138(42.9)$ & $76(42.9)$ \\
\hline Presence of CHD, cases (\%) & $58(18.0)$ & $37(20.9)$ \\
\hline Presence of CKD, cases $\%)$ & $43(13.4)$ & $13(7.3)$ \\
\hline Presence of stroke, case (\%) & $184(57.1)$ & $85(48.0)$ \\
\hline Undergoing medication, cases (\%) & $244(75.8)$ & $139(78.5)$ \\
\hline Smoker $(\%)$ & $111(34.5)$ & $3(1.7)$ \\
\hline Drinker $(\%)$ & $53(16.5)$ & $2(1.1)$ \\
\hline
\end{tabular}

Table 1. Clinical characteristics of subjects. Continuous variables were presented as means \pm standard deviation (SD) and categorical data were presented as the number (percentage). Differences between groups were examined by using T test or $\chi 2$ tests according to the characteristics of data distribution. BMI body mass index, $H C Y$ homocysteine, $H b A 1 c$ hemoglobin A1c, BUN blood urea nitrogen, TC total cholesterol, $T G$ triglyceride, $H D L-C$ high density lipoprotein cholesterol, $L D L-C$ low density lipoprotein cholesterol, $S B P$ systolic blood pressure, $D B P$ diastolic blood pressure, $L V E F$ left ventricular ejection fraction, $H T N$ hypertension, $C H D$ coronary heart disease, $C K D$ chronic kidney disease.

and HCY, TC, TG and LDL-C levels $(P=0.087)$ were factors positively associated with serum uric acid levels in male patient with hypertension; serum uric acid levels were higher in those with CKD and stroke and lower in those complicated with diabetes $(P=0.069)$. Age and HCY levels were positively associated and HDL-C levels $(P=0.086)$ and LVEF were negatively associated with serum uric acid levels in female patients; serum uric acid levels were higher in females complicated with CKD and/or stroke.

Selection of variables for the multivariate regression analysis. Strict quality control is one of the preconditions to ensure the reliability of multivariate analysis. In this report, the principles of biochemistry, clinical practice and Pearson correlation analyses were adopted comprehensively to be the basis for variable selection.

In general, serum creatinine, BUN and uric acid levels solely acted as dependent variables; HDL-C and LDL-C were selected to represent lipid profile; "SBP and DBP" and "the presence of diabetes and undergoing glycemic control" were input into the multivariate regression model individually to avoid multicollinearity.

Thus, "HCY, LDL-C, SBP/DBP and LVEF" and "age, HCY, presence of CHD, CKD and stroke" were variable sets for males and females in the multivariate regression analysis to identify the independent associations with serum creatinine levels. Age, HCY and TC levels, LVEF, undergoing glycemic control, the presence of CKD and stroke, and alcohol consumption were variable sets for males, and age, HCY and LDL-C levels, DBP, LVEF, undergoing glycemic control, the presence of $\mathrm{CHD}, \mathrm{CKD}$ and stroke were variable sets for females to identify the independent associations with serum BUN levels. Age, BMI, HCY, HbA1c, LDL-C, and HDL-C levels and the presence of diabetes, CKD and stroke were variable sets for males, and age, HCY and HDL-C levels, LVEF, and the presence of CKD and stroke were variable sets for females to identify independent associations with serum uric acid levels.

Factors independently associated with serum creatinine levels. As shown in Table 3, multivariate regression analyses showed that HCY and LDL-C levels were factors positively associated and LVEF was a factor negatively associated with serum creatinine levels independently in males with hypertension. Neither SBP nor DBP were independent factors associated with serum creatinine levels in males. In females with hypertension, 


\begin{tabular}{|c|c|c|c|c|}
\hline \multirow[b]{2}{*}{ Variable } & \multicolumn{2}{|l|}{ Male } & \multicolumn{2}{|l|}{ Female } \\
\hline & $(\mathrm{N}=323)$ & $P$ & $(\mathrm{~N}=177)$ & $P$ \\
\hline \multicolumn{5}{|c|}{ Factors associated with serum creatinine level } \\
\hline Age, years & - & - & $1.29(0.67$ to 1.90$)$ & $<0.001$ \\
\hline $\mathrm{HCY}, \mu \mathrm{mol} / \mathrm{L}$ & $2.59(1.51$ to 3.67$)$ & $<0.001$ & $2.74(1.57$ to 3.92$)$ & $<0.001$ \\
\hline $\mathrm{BUN}, \mathrm{mmol} / \mathrm{L}$ & $15.33(13.74$ to 16.92$)$ & $<0.001$ & $13.48(12.02$ to 14.95$)$ & $<0.001$ \\
\hline Uric acid, $\mu \mathrm{mol} / \mathrm{L}$ & $0.10(0.03$ to 0.18$)$ & 0.007 & $0.21(0.14$ to 0.27$)$ & $<0.001$ \\
\hline $\mathrm{TC}, \mathrm{mmol} / \mathrm{L}$ & $10.21(2.72$ to 17.70$)$ & 0.008 & - & - \\
\hline $\mathrm{LDL}-\mathrm{C}, \mathrm{mmol} / \mathrm{L}$ & $10.58(2.01$ to 19.15$)$ & 0.016 & - & - \\
\hline SBP, mmHg & $0.43(0.09$ to 0.77$)$ & 0.013 & - & - \\
\hline $\mathrm{DBP}, \mathrm{mmHg}$ & $0.60(-0.60$ to 1.26$)$ & 0.074 & - & - \\
\hline LVEF, \% & $-0.33(-0.46$ to -0.19$)$ & & - & - \\
\hline Presence of CHD, cases (\%) & - & - & $1.01(1.00$ to 1.01$)$ & 0.099 \\
\hline Presence of CKD, cases (\%) & - & - & $1.10(1.04$ to 1.16$)$ & $<0.001$ \\
\hline Presence of stroke, case (\%) & - & - & $0.99(0.98$ to 1.00$)$ & 0.046 \\
\hline \multicolumn{5}{|c|}{ Factors associated with serum BUN } \\
\hline Age, years & $0.05(0.02$ to 0.07$)$ & 0.001 & $0.09(0.05$ to 0.12$)$ & $<0.001$ \\
\hline $\mathrm{HCY}, \mu \mathrm{mol} / \mathrm{L}$ & $0.12(0.07$ to 0.17$)$ & $<0.001$ & $0.19(0.13$ to 0.27$)$ & $<0.001$ \\
\hline $\mathrm{TC}, \mathrm{mmol} / \mathrm{L}$ & $0.31(-0.05$ to 0.67$)$ & 0.091 & - & - \\
\hline LDL-C, mmol/L & - & - & $-0.37(-0.79$ to 0.06$)$ & 0.093 \\
\hline $\mathrm{DBP}, \mathrm{mmHg}$ & - & - & $-0.04(-0.08$ to 0.00$)$ & 0.076 \\
\hline LVEF, \% & $-0.09(-0.16$ to -0.01$)$ & 0.018 & $-0.12(-0.21$ to -0.36$)$ & 0.006 \\
\hline Diabetes, cases (\%) & $1.17(1.08$ to 1.27$)$ & $<0.001$ & - & - \\
\hline Diabetes treatment, cases (\%) & $1.16(1.07$ to 1.25$)$ & $<0.001$ & $1.12(1.00$ to 1.24$)$ & 0.043 \\
\hline Presence of CHD, cases (\%) & - & - & $1.11(1.00$ to 1.24$)$ & 0.056 \\
\hline Presence of CKD, cases (\%) & 1.55 (1.36 to 1.77$)$ & $<0.001$ & $1.75(1.38$ to 2.21$)$ & $<0.001$ \\
\hline Presence of stroke, case (\%) & $0.94(0.88$ to 1.01$)$ & 0.076 & $0.86(0.75$ to 0.97$)$ & 0.016 \\
\hline Drinker (\%) & $0.87(0.76$ to 0.99$)$ & 0.032 & - & - \\
\hline \multicolumn{5}{|c|}{ Factors associated with serum uric acid } \\
\hline Age, years & $-0.71(-1.54$ to 0.12$)$ & 0.093 & $1.47(0.11$ to 2.83$)$ & 0.035 \\
\hline BMI, $\mathrm{kg} / \mathrm{m}^{2}$ & $6.63(92.81$ to 10.45$)$ & 0.001 & & \\
\hline $\mathrm{HCY}, \mu \mathrm{mol} / \mathrm{L}$ & $2.28(0.69$ to 3.87$)$ & 0.005 & $3.42(0.81$ to 6.02$)$ & 0.011 \\
\hline HbAlc, \% & $-0.10(-0.17$ to -0.04$)$ & 0.002 & - & - \\
\hline $\mathrm{TC}, \mathrm{mmol} / \mathrm{L}$ & $11.31(0.48$ to 22.14$)$ & 0.041 & - & - \\
\hline $\mathrm{TG}, \mathrm{mmol} / \mathrm{L}$ & $13.59(2.43$ to 24.75$)$ & 0.017 & - & - \\
\hline $\mathrm{LDL}-\mathrm{C}, \mathrm{mmol} / \mathrm{L}$ & $10.81(-1.59$ to 23.21$)$ & 0.087 & - & - \\
\hline $\mathrm{HDL}-\mathrm{C}, \mathrm{mmol} / \mathrm{L}$ & $-51.96(-91.49$ to -12.43$)$ & 0.011 & $-39.14(-83.88$ to 5.60$)$ & 0.086 \\
\hline LVEF, \% & - & - & $-3.98(-7.11$ to -0.86$)$ & 0.013 \\
\hline Presence of diabetes, cases (\%) & $0.99(0.99$ to 1.00$)$ & 0.069 & - & - \\
\hline Presence of CKD, cases (\%) & $1.01(1.01$ to 1.01$)$ & $<0.001$ & $1.01(1.01$ to 1.02$)$ & $<0.001$ \\
\hline Presence of stroke, case (\%) & $1.00(1.00$ to 1.00$)$ & 0.012 & $1.00(0.99$ to 1.00$)$ & 0.019 \\
\hline
\end{tabular}

Table 2. Factors associated with renal function in the univariate regression analysis. For the abbreviations, please see Table 1. "-" indicates that a variable is not a potential independent one in a given univariate analysis model $(\mathrm{p}>0.1)$. HCY was input into each model as continuous variable. When both dependent and independent variates were continuous variables, the associations were quantified by coefficients and $95 \%$ confidential intervals (95\% CIs); when dependent variables were categorical variable, the associations were quantified by odds ratios (ORs) and $95 \%$ CIs.

only age and the presence of CKD remained as independent factors to predict serum creatinine levels; HCY levels, the presence of $\mathrm{CHD}$ and stroke lost independence in association with serum creatinine levels in females.

Factors independently associated with serum BUN levels. As shown in Table 4, multivariate regression analyses showed that age, HCY and TC levels, undergoing glycemic control and the presence of CKD were positively associated with serum BUN levels in males with hypertension. LVEF, the presence of stroke and alcohol consumption were not independent factors to predict serum BUN levels in males. Age and the presence of CKD were positively associated and LDL-C levels and LVEF were negatively associated with serum BUN levels in females with hypertension. HCY levels, DBP, undergoing glycemic control, and the presence of CHD and stroke were not independent risk factors associated with serum BUN levels in females with hypertension. 


\begin{tabular}{|l|l|l|l|l|}
\hline \multirow{2}{*}{ Variable } & Male & \multicolumn{2}{l|}{ Female } \\
\cline { 2 - 5 } & $\mathbf{( N = 3 2 3 )}$ & $\boldsymbol{P}$ & $\mathbf{( N = 1 7 7 )}$ & $\boldsymbol{P}$ \\
\hline Age, years & - & & $0.48(0.01$ to 0.95$)$ & 0.043 \\
\hline HCY, $\mu \mathrm{mol} / \mathrm{L}$ & $2.04(1.08$ to 3.00$)$ & $<0.001$ & - & - \\
\hline LDL-C, $\mathrm{mmol} / \mathrm{L}$ & $10.26(3.05$ to 17.48$)$ & 0.005 & - & - \\
\hline LVEF, \% & $-3.00(-4.32$ to -1.70$)$ & $<0.001$ & - & - \\
\hline Presence of CKD, cases (\%) & - & - & $127.39(107.20$ to 147.57$)$ & $<0.001$ \\
\hline
\end{tabular}

Table 3. Factors associated with serum creatinine levels in the multivariate regression analysis. For the abbreviations, please see Table 1 . Associations are expressed as coefficients and 95\% CIs. "-" indicates that a variable is not an independent one in a given multivariate analysis model $(\mathrm{p}>0.05)$.

\begin{tabular}{|l|l|l|l|l|}
\hline \multirow{2}{*}{ Variable } & \multicolumn{2}{|l|}{ Male } & Female \\
\cline { 2 - 5 } & $\mathbf{( N = 3 2 3 )}$ & $\boldsymbol{P}$ & $\mathbf{N}=\mathbf{1 7 7})$ & $\boldsymbol{P}$ \\
\hline Age, years & $0.05(0.02$ to 0.07$)$ & $<0.001$ & $0.04(0.01$ to 0.07$)$ & 0.025 \\
\hline HCY, $\mu \mathrm{mol} / \mathrm{L}$ & $0.07(0.02$ to 0.11$)$ & 0.006 & - & - \\
\hline TC, $\mathrm{mmol} / \mathrm{L}$ & $0.61(0.30$ to 0.93$)$ & $<0.001$ & - & - \\
\hline LDL-C, $\mathrm{mmol} / \mathrm{L}$ & - & - & $-0.39(-0.70$ to -0.08$)$ & 0.016 \\
\hline LVEF, $\%$ & - & - & $-0.10(-0.16$ to -0.03$)$ & 0.004 \\
\hline Diabetes treatment, cases $(\%)$ & $1.46(0.81$ to 2.11$)$ & $<0.001$ & - & - \\
\hline Presence of CKD, cases $(\%)$ & $3.89(2.94$ to 4.83$)$ & $<0.001$ & $6.15(4.71$ to 7.60$)$ & $<0.001$ \\
\hline
\end{tabular}

Table 4. Factors associated with BUN levels in the multivariate regression analysis. For the abbreviations, please see Table 1. Associations are expressed as coefficients and 95\% CIs. "-" indicates that a variable is not an independent one in a given multivariate analysis model $(\mathrm{p}>0.05)$.

\begin{tabular}{|l|l|l|l|l|}
\hline \multirow{2}{*}{ Variable } & \multicolumn{2}{|l|}{ Male } & Female \\
\cline { 2 - 5 } & $(\mathbf{N}=\mathbf{3 2 3})$ & $\boldsymbol{P}$ & $\mathbf{( N = 1 7 7 )}$ & $\boldsymbol{P}$ \\
\hline BMI & $5.59(1.82$ to 9.36$)$ & 0.004 & - & - \\
\hline HbAlc, \% & $-0.13(-0.20$ to -0.06$)$ & $<0.001$ & - & - \\
\hline HDL-C, mmol/L & $-0.50(-0.88$ to -0.13$)$ & 0.008 & - & - \\
\hline LVEF, \% & - & - & $-4.03(-7.02$ to -1.05$)$ & 0.008 \\
\hline Presence of CKD, cases (\%) & $80.20(48.13$ to 112.27$)$ & $<0.001$ & $108.85(50.25$ to 167.46$)$ & $<0.001$ \\
\hline Presence of stroke, case (\%) & $-25.50(-47.24$ to -3.76$)$ & 0.022 & - & - \\
\hline
\end{tabular}

Table 5. Factors associated with serum uric acid levels in the multivariate regression analysis. For the abbreviations, please see Table 1 . Associations are expressed as coefficients and 95\% CIs. "-" indicates that a variable is not an independent one in a given multivariate analysis model $(p>0.05)$.

Factors independently associated with serum uric acid levels. As shown in Table 5, when age, BMI, $\mathrm{HCY}, \mathrm{HbAlc}$, LDL-C, and HDL-C levels and the presence of diabetes, CKD and stroke were inputted into the multivariable regression model, only $\mathrm{BMI}$ and presence of $\mathrm{CKD}$ remained as independent risk factors positively associated and HbAlc and HDL-C levels and the presence of stroke remained as independent risk factors negatively associated with serum uric acid levels in males with hypertension. Of females with hypertension, among the potential risk factors, age, HCY and HDL-C levels, LVEF, the presence of CKD and stroke, only LVEF and presence of CKD remained as independent predictors that were negatively and positively associated with serum uric acid levels.

\section{Discussion}

Our data showed that HCY levels were independent predictors positively associated with increased serum creatinine and BUN levels only in male patients. Since the radioenzymic determination showed that only tiny amounts of homocysteine are excreted in the urine ${ }^{3,25}$ and serum creatinine and BUN levels are the two main parameters that reflect renal capacity to eliminate metabolic byproducts, the higher serum homocysteine, the higher creatinine and BUN levels in male patients with hypertension should be caused by distinct mechanisms. Most likely, the former is caused by impaired nonrenal disposal, and the latter is a consequence of reduced renal elimination. Together with the biotoxicity of homocysteine described in the "Introduction"1, disorders in homocysteine metabolism might cause direct damage to the kidneys. Studies have showed that imbalance in homocysteine metabolism lead to molecular and cellular damage on many organs via homocysteinylation, 
oxidative stress induction, and excitotoxicity ${ }^{1-3,7,19}$. High homocysteine level in old male patients with hypertension may also be harmful to renal corpuscles, renal tubules and renal artery through the above mechanism. On the other hand, high circulating homocysteine levels might also lead to secondary renal injury via other complications such as hypertension ${ }^{2}$, etc.

In terms of why the above results were not observed in females with hypertension, numerous reports are consistent with our results and provide possible mechanism ${ }^{26,27}$. Firstly, different sex hormone levels between men and women may affect the metabolism of homocysteine ${ }^{26,27}$; the Third National Health and Nutrition Examination Survey stated that "...higher estrogen status is associated with a decreased mean serum total homocysteine concentration..." ${ }^{28,29}$. Secondly, the serum homocysteine concentration in smokers is higher than that in nonsmokers $^{30}$; in our subjects, $34.5 \%$ males smoke, in contrast, only $1.7 \%$ females smoke. Finally, the level of the homocysteine in the serum is influenced by the presence of folic acid, vitamins B6 and B12 $2^{31}$; the folic acid level of our female subjects is $9.2 \pm 4.7 \mathrm{ng} / \mathrm{mL}$, which is higher than that of males $(7.6 \pm 4.6 \mathrm{ng} / \mathrm{mL})$.

Different from serum creatinine and BUN levels, serum uric acid levels are determined by the balance between dietary intake, endogenous metabolism of purines and the urinary excretion rate $^{32}$; higher serum uric acid levels are always caused by overeating. Homocysteine is neither an independent risk factor for uric acid in males nor in females, which suggests that homocysteine is associated with renal damage through a pathway different from that involved in metabolic disorders.

The serum lipid representative LDL-C was an independent risk factor that was positively associated with increased serum creatinine levels only in male patients and negatively associated with increased serum BUN levels only in female patients. Although this content was not the focus of this study, the research available for reference is also very limited ${ }^{33}$. LDL-C levels were reported to be lower or higher in CKD patients ${ }^{34,35}$; since high LDL-C levels are a cause of atherosclerosis, it is understandable that LDL-C is positively associated with serum creatinine levels. What is puzzling is that LDL-C was negatively associated with BUN levels in female patients. A possible explanation for these results is sex differences and a discrepancy in LDL-C and BUN in their respective metabolic pathways. Reduced protein intake in our female subjects might be a reason why the higher the LDL-C levels were, the lower the BUN levels. HDL-C levels were independent risk factors that were negatively associated with increased serum uric acid levels only in male patients. Studies have shown that lower plasma apoA-I and plasma lecithin-cholesterol acyltransferase activity are present in subjects with chronic renal failure and lead to impaired HDL-mediated reverse cholesterol transport ${ }^{33,36,37}$. Although only $13.4 \%$ of male patients and $7.3 \%$ of female patients had CKD, the above mechanism could partially explain the associations of HDL-C in our report. In addition, negative associations between HDL-C and uric acid levels were also observed in asymptomatic dyslipidemic subjects ${ }^{38}$, which is also partially consistent with our results.

Age was a common independent risk factor that was positively associated with serum BUN levels in both sexes and was independently associated with serum creatinine levels in female patients. Age and sex are known to be two critical factors to estimate the glomerular filtration rate ${ }^{39}$; thus, the fact that age is associated with renal function is consistent with existing scientific knowledge. In terms of why age is not an independent factor associated with serum creatinine levels in males, this might be due to the particularity of age composition in our subjects between sexes.

LVEF was independently and negatively associated with serum BUN and uric acid levels in female patients and serum creatinine levels in male patients. Renal function disorders share many risk factors for CHD and hypertension ${ }^{40}$; thus, although the associations differed by sex and renal function indicators, our data suggest that the higher the serum creatinine, BUN and uric acid levels are, the lower the LVEF, which is in line with biomedical principles.

Regarding medical history, glycemic control was positively associated and the presence of stroke was negatively associated with serum BUN and uric acid levels in males with hypertension. The lower uric acid levels in male patients with hypertension and stroke might be due to diet control among these subjects. Other variables, such as smoking, alcohol consumption and taking medications for hypertension and/or CHD were not associated with renal function in males even in the preliminary univariate regression analyses; this might be due to the relatively low role of these factors in renal damage in older patients with hypertension.

In addition to acting as a renal function indicator, serum uric acid levels are also a main parameter for metabolic disorders ${ }^{41}$. BMI and HbA1c emerged as risk factor that were independently associated with serum uric acid levels in males with hypertension, which is in line with the above principles and the metabolic characteristics of our subjects that were summarized in the Table 1.

It is worth emphasizing that our study subjects were older Chinese adults with hypertension; our analytic strategy treated renal function indicators as continuous variables; only $13.4 \%$ of males and $7.3 \%$ of females were diagnosed with CKD, and our study quantified the relationship between biochemical indexes, including homocysteine levels and renal function but not the presence of CKD in adults with hypertension. Since hypertension, CKD, stroke and CHD are highly prevalent in older Chinese individuals, it is impossible to assemble enough age-matched and complication-free controls to rule out the association between homocysteine levels and renal function or hypertension in clinical practice.

In conclusion, our data indicate that in older Chinese males with hypertension, homocysteine levels are independent risk factors that are positively associated with increased serum creatinine and urea nitrogen levels. Our data imply that clinicians need to be aware of any possible clinical and subclinical renal injury caused by higher homocysteine level in old males with hypertension. Longitudinal cohort study is needed to evaluate a causal relationship between higher homocysteine level and renal damage in this population. Also, study to evaluate whether lowering homocysteine intervention (e.g. using folic acid and B vitamins) could improve renal function in older males with hypertension has clinical significance. 
Received: 15 August 2020; Accepted: 9 October 2020

Published online: 22 October 2020

\section{References}

1. Škovierová, H. et al. The molecular and cellular effect of homocysteine metabolism imbalance on human health. Int. J. Mol. Sci. 17, 1733, https://doi.org/10.3390/ijms17101733 (2016).

2. Cianciolo, G. et al. Folic acid and homocysteine in chronic kidney disease and cardiovascular disease progression: Which comes first?. Cardiorenal Med. 7, 255-266 (2017).

3. van Guldener, C. \& Robinson, K. Homocysteine and renal disease. Semin. Thromb. Hemost. 26, 313-324 (2000).

4. Capelli, I. et al. Folic acid and vitamin B12 administration in CKD, why not? Nutrients 11, 383, https://doi.org/10.3390/nu110 20383 (2019).

5. Francis, M. E., Eggers, P. W., Hostetter, T. H. \& Briggs, J. P. Association between serum homocysteine and markers of impaired kidney function in adults in the United States. Kidney Int. 66, 303-312 (2004).

6. Elhawary, N. A. et al. The MTHFR 677T allele may influence the severity and biochemical risk factors of Alzheimer's disease in an Egyptian population. Dis. Markers 35, 439-446 (2013).

7. Lai, W. K. \& Kan, M. Y. Homocysteine-induced endothelial dysfunction. Ann. Nutr. Metab. 67, 1-12 (2015).

8. Thompson, S. et al. Cause of death in patients with reduced kidney function. J. Am. Soc. Nephrol. 26, 2504-2511 (2015).

9. Gu, P. et al. Relationship between serum homocysteine levels and depressive symptoms: The Cooper Center Longitudinal Study. J. Clin. Psychiatry 73, 691-695 (2012).

10. Schalinske, K. L. \& Smazal, A. L. Homocysteine imbalance: A pathological metabolic marker. Adv. Nutr. 3, 755-762 (2012).

11. Givvimani, S. et al. Hyperhomocysteinemia decreases intestinal motility leading to constipation. Am. J. Physiol. Gastrointest. Liver Physiol. 303, G281-290 (2012).

12. Zappacosta, B., Persichilli, S., Minucci, A., Giardina, B. \& Maurizi, M. Serum levels of folate, homocysteine, and vitamin B12 in head and neck squamous cell carcinoma and in laryngeal leukoplakia. Cancer 103, 284-292 (2005).

13. Iacobazzi, V., Infantino, V., Castegna, A. \& Andria, G. Hyperhomocysteinemia: Related genetic diseases and congenital defects, abnormal DNA methylation and newborn screening issues. Mol. Genet. Metab. 113, 27-33 (2014).

14. Cohen, E., Margalit, I., Shochat, T., Goldberg, E. \& Krause, I. The relationship between the concentration of plasma homocysteine and chronic kidney disease: A cross sectional study of a large cohort. J. Nephrol. 32, 783-789 (2019).

15. Ninomiya, T. et al. Hyperhomocysteinemia and the development of chronic kidney disease in a general population: The Hisayama study. Am. J. Kidney Dis. 44, 437-445 (2004).

16. Jager, A. et al. Serum homocysteine levels are associated with the development of (micro)albuminuria: The Hoorn study. Arterioscler. Thromb. Vasc. Biol. 21, 74-81 (2001).

17. Chuang, C. H. et al. Homocysteine and C-reactive protein as useful surrogate markers for evaluating CKD risk in adults. Kidney Blood Press. Res. 37, 402-413 (2013).

18. Choi, S. T., Kim, J. S. \& Song, J. S. Elevated serum homocysteine levels were not correlated with serum uric acid levels, but with decreased renal function in gouty patients. J. Korean Med. Sci. 29, 788-792 (2014).

19. Gao, N. et al. Low doses of folic acid can reduce hyperhomocysteinemia-induced glomerular injury in spontaneously hypertensive rats. Hypertens. Res. https://doi.org/10.1038/s41440-020-0471-8 (2020).

20. Tao, L. X. et al. Association between plasma homocysteine and hypertension: Results from a cross-sectional and longitudinal analysis in Beijing's adult population from 2012 to 2017. J. Clin. Hypertens. (Greenwich) 20, 1624-1632 (2018).

21. Ye, Z. et al. Prevalence of homocysteine-related hypertension in patients with chronic kidney disease. J. Clin. Hypertens. (Greenwich) 19, 151-160 (2017).

22. Huo, Y. et al. Efficacy of folic acid therapy in primary prevention of stroke among adults with hypertension in China: The CSPPT randomized clinical trial. JAMA 313, 1325-1335 (2015).

23. Knuuti, J. et al. 2019 ESC Guidelines for the diagnosis and management of chronic coronary syndromes. Eur. Heart J. 41, 407-477 (2020).

24. American Diabetes Association. 2. Classification and diagnosis of diabetes: Standards of medical care in diabetes-2020. Diabetes Care 43, S14-S31 (2020).

25. Refsum, H., Helland, S. \& Ueland, P. M. Radioenzymic determination of homocysteine in plasma and urine. Clin. Chem. 31, 624-628 (1985).

26. Mohan, I. K. et al. Application of adaptive neuro-fuzzy inference systems (ANFIS) to delineate estradiol, glutathione and homocysteine interactions. Clin. Nutr. ESPEN 20, 41-46 (2017).

27. Cohen, E., Margalit, I., Shochat, T., Goldberg, E. \& Krause, I. Gender differences in homocysteine concentrations, a populationbased cross-sectional study. Nutr. Metab. Cardiovas. Dis. (NMCD). 29, 9-14. https://doi.org/10.1016/j.numecd.2018.09.003 (2019).

28. Dimitrova, K. R., DeGroot, K., Myers, A. K. \& Kim, Y. D. Estrogen and homocysteine. Cardiovasc. Res. 53, 577-588. https://doi. org/10.1016/s0008-6363(01)00462-x (2002).

29. Morris, M. S., Jacques, P. F., Selhub, J. \& Rosenberg, I. H. Total homocysteine and estrogen status indicators in the Third National Health and Nutrition Examination Survey. Am. J. Epidemiol. 152, 140-148. https://doi.org/10.1093/aje/152.2.140 (2000).

30. O'Callaghan, P., Meleady, R., Fitzgerald, T., Graham, I. \& European COMAC group. Smoking and plasma homocysteine. Eur. Heart J. 23, 1580-1586, https://doi.org/10.1053/euhj.2002.3172 (2002).

31. Zaric, B. L. et al. Homocysteine and hyperhomocysteinaemia. Curr. Med. Chem. 26, 2948-2961. https://doi.org/10.2174/09298 67325666180313105 (2019).

32. Kutzing, M. K. \& Firestein, B. L. Altered uric acid levels and disease states. J. Pharmacol. Exp. Ther. 324, 1-7 (2008).

33. Hager, M. R., Narla, A. D. \& Tannock, L. R. Dyslipidemia in patients with chronic kidney disease. Rev. Endocr. Metab. Disord. 18, $29-40$ (2017).

34. Oi, K., Hirano, T., Sakai, S., Kawaguchi, Y. \& Hosoya, T. Role of hepatic lipase in intermediate-density lipoprotein and small, dense low-density lipoprotein formation in hemodialysis patients. Kidney Int. Suppl. 71, S227-228 (1999).

35. Ikewaki, K. et al. Delayed in vivo catabolism of intermediate-density lipoprotein and low-density lipoprotein in hemodialysis patients as potential cause of premature atherosclerosis. Arterioscler. Thromb. Vasc. Biol. 25, 2615-2622 (2005).

36. Vaziri, N. D., Deng, G. \& Liang, K. Hepatic HDL receptor, SR-B1 and Apo A-I expression in chronic renal failure. Nephrol. Dial. Transplant. 14, 1462-1466 (1999).

37. Vaziri, N. D., Liang, K. \& Parks, J. S. Down-regulation of hepatic lecithin:cholesterol acyltransferase gene expression in chronic renal failure. Kidney Int. 59, 2192-2196 (2001).

38. Cibičková, L, Langová, K., Vaverková, H., Kubíčková, V. \& Karásek, D. Correlation of uric acid levels and parameters of metabolic syndrome. Physiol. Res. 66, 481-487 (2017).

39. Levey, A. S. \& Inker, L. A. Assessment of glomerular filtration rate in health and disease: A state of the art review. Clin. Pharmacol. Ther. 102, 405-419 (2017).

40. Borghi, C. et al. Serum uric acid and the risk of cardiovascular and renal disease. J. Hypertens. 33, 1729-1741 (2015).

41. King, C. et al. Uric acid as a cause of the metabolic syndrome. Contrib. Nephrol. 192, 88-102 (2018). 


\section{Author contributions}

Q.Y. conceived the study, designed the experiments, supervised the project, and wrote the manuscript. Q.Y., Y.L., Y.D., J.X. and X.Z. performed the experiments, analyzed the results, and wrote the manuscript. All authors had full access to all of the data in the study and can take responsibility for the integrity of the data and the accuracy of the data analysis.

\section{Competing interests}

The authors declare no competing interests.

\section{Additional information}

Correspondence and requests for materials should be addressed to Q.Y.

Reprints and permissions information is available at www.nature.com/reprints.

Publisher's note Springer Nature remains neutral with regard to jurisdictional claims in published maps and institutional affiliations.

(c) (i) Open Access This article is licensed under a Creative Commons Attribution 4.0 International License, which permits use, sharing, adaptation, distribution and reproduction in any medium or format, as long as you give appropriate credit to the original author(s) and the source, provide a link to the Creative Commons licence, and indicate if changes were made. The images or other third party material in this article are included in the article's Creative Commons licence, unless indicated otherwise in a credit line to the material. If material is not included in the article's Creative Commons licence and your intended use is not permitted by statutory regulation or exceeds the permitted use, you will need to obtain permission directly from the copyright holder. To view a copy of this licence, visit http://creativecommons.org/licenses/by/4.0/.

(C) The Author(s) 2020 